\title{
HLF is a Potential Prognostic Biomarker in Head and Neck Squamous Cell Carcinoma Based on Bioinformatic Analysis and Experimental Validati on
}

\author{
Wei Fang \\ Stomatology Hospital,Southern Medical University \\ Di Wan \\ Stomatology Hospital,Southern Medical University \\ Jun Chen \\ Stomatology Hospital,Southern Medical University \\ Weiqun Ma \\ Stomatology Hospital,Southern Medical University \\ Zhen Luo
}

Stomatology Hospital,Southern Medical University

Xiaoqin Yang ( $\square$ yangxqkq@163.com )

Stomatology Hospital,Southern Medical University https://orcid.org/0000-0002-0842-0531

\section{Research}

Keywords: Differentially expressed genes (DEGs), Gene Expression Omnibus (GEO), Bioinformatics, HLF, Biomarker

Posted Date: December 30th, 2020

DOI: https://doi.org/10.21203/rs.3.rs-136194/v1

License: (c) (i) This work is licensed under a Creative Commons Attribution 4.0 International License. Read Full License 


\section{Abstract}

\section{Background}

Head and neck squamous cell carcinoma (HNSCC) is one of the most frequent cancers worldwide, with an increasing incidence. However, the underlying molecular mechanisms of HNSCC are poorly understood.

\section{Method}

In this work, 5 original datasets (GSE23558, GSE13601, GSE30784, GSE9844, GSE78060) of Head and neck squamous cell carcinoma (HNSCC) were selected from Gene Expression Omnibus (GEO) database. To identify differentially expressed genes (DEGs) in HNSCC and adjacent tissues. The common DEGs were acquired by Venn diagram. The sensitivity and specificity of HLF were determined by Receiver operating characteristic curves (ROC). Then, In order to further confirm the relationship between HLF and HNSCC patient's prognosis, the expression and survival analysis of HLF was performed by Gene Expression Profiling Interactive Analysis (GEPIA), Cell culture, reverse transcription polymerase chain reaction (RT-PCR), western blotting and immunohistochemical staining.

\section{Results}

Seventeen DEGs were screened from five sets of HNSCC functional gene expression series in GEO datasets. The low expression of HLF was indicated might be correlated with poor prognosis of HNSCC patients based on the bioinformatics analysis. According to the results of Cell culture, RT-PCR, western blotting, immunohistochemical staining, it was confirmed that the low level of HLF expression correlated with poor prognosis of HNSCC patients.

\section{Conclusion}

The study effectively revealed useful information about the relationship of the low level of HLF expression and HNSCC. In summary, we identified HLF as a potential prognostic biomarker and therapeutic target for HNSCC.

\section{Introduction}

Head and neck squamous cell carcinoma (HNSCC) is one of the most common types of cancer worldwide, with $>450,000$ patients diagnosed every year[1]. Despite the integrated application of these therapies, the overall 5-year survival rate of HNSCC patients is $<50 \%$ [2-3]. Although distinctive advances in the field of HNSCC biomarker discovery, no clinically useful potential prognostic biomarker have been found yet for HNSCC [4].

It is well understood that tumor development is a multi-factorial, multi-stage process, even the same tumor that occurs in the same patient but in different lesion areas or different disease stages may 
express different molecules[5]. Comprehensive studies are needed to provide the basis for identification of the reliable prognostic biomarker. The differentially expressed genes (DEGs) or differentially expressed proteins (DEPS) can be identified between tumor and normal tissues by bioinformatics methods, and further analyzed the correlation between patients survival rate and DEGs or DEPs, so as to screen the potential tumor prognostic markers. Some scholars considered that DDB2 (damaged DNA binding protein 2) had enhanced the expression of angiogenic markers and promotes tumor growth in a xenograft model, and had a significant suppressive effect on expression of the endogenous markers of hypoxia that were also prognostic indicators in HNSCC[6]. Zhao et al [7] identified 9 hub genes((SPP1, ITGA6, TMPRSS11D, MMP1, LAMC2, FAT1, ACTA1, SERPINE1 and CEACAM1) significantly correlated with HNSCC, and considered that these nine hub genes may serve as potential tumor markers and important prognostic indicators for HNSCC. However, these results remain controversial. Another study concerning HNSCC found no correlation between SPP1 expression level and prognosis of HNSCC [8]. Therefore, more valuable prognostic markers need to be identified.

In the present study, 5 original datasets (GSE23558, GSE13601, GSE30784, GSE9844, GSE78060) from Gene Expression Omnibus (GEO) database (https://www.ncbi.nlm.nih.gov/geo) were downloaded and analyzed in order to select DEGs between HNSCC and normal tissues. 17 common differentially expressed genes (DEGs) were identified between HNSCC tissues and normal controls from 5 independent GEO datasets. The clinical data in the TCGA database also confirmed the effect of low expression of HLF might be correlated with poor prognosis and patient survival. RT-PCR, western blotting and immunohistochemical staining were carried out to reveal the relationship between the expression level of HLF and HNSCC patient's prognosis.

\section{Materials And Methods}

\section{HNSCC DEGs and prognostic biomarker screening by bioinformatics}

Five GEO Series (GSE23558,32 samples; GSE13601ه58 samples; GSE30784囚229 samples; GSE9844₫38 samples; GSE78060®30 samples) were analyzed separately using online GEO2R with default parameters (https://www.ncbi.nlm.nih.gov/geo/geo2r/). The DEGs with the cutoff criteria of /the $\log _{2}$-fold change $(\log F C) \mid>2$ and adj.P.value $\leq 0.05$ were considered to be significantly different. Venn diagram to determine common HNSCC DEGs of these five series was prepared using the online application (http://bioinformatics.psb.ugent.be/webtools/Venn/). Subsequently, the common genes were defined as hub genes if they were highly correlated with HNSCC. In addition, the sensitivity and specificity of HLF as a prognostic biomarker were determined by Receiver operating characteristic curves (ROC).

In order to confirm and increase the reliability of the data analysis,the expression and survival analysis of HLF was performed using Gene Expression Profiling Interactive Analysis (GEPIA, http://gepia.cancerpku.cn/), an online analysis software based on the TCGA and Genotype-Tissue Expression (GTEx) databases. The results were shown as box plots and Kaplan-Meier survival curves[9]. 


\section{Experimental identification of the prognostic biomarker HLF for HNSCC}

The ethical committee of the Stomatological Hospital, Southern Medical University approved the present study. All participants were asked to provide signed informed consent to participate in the trial. The present study was performed in accordance with the guidelines of the World Medical Association Declaration of Helsinki Ethical Principles. All the samples of TSCC were collected from the Stomatological Hospital, Southern Medical University. No patient had received radiotherapy or chemotherapy

prior to operation.

\section{Immunohistochemistry}

This is a prospective study conducted between February 2008 and December 2015. A total of 64 cases met inclusion criteria. The inclusion criteria were as follows: (1) the maximum diameter of primary tumors $\leq 3 \mathrm{~cm}$; (2) All surgeries were done by the same surgeon. Sections made of archived FFPE tissues were subjected to both HE staining and immunohistochemical staining.

The sections were soaked in $3 \%$ hydrogen peroxide for 10 min to block endogenous peroxidase activity and treated with ethylenediamine tetraacetic acid (EDTA) buffer $(\mathrm{pH}=8.0)$ for antigen retrieval. Then, the sections were treated with $1 \%$ bovine serum to block nonspecific background binding. Anti HLF antibody ( $1 \mathrm{mg} / \mathrm{mL}$; Thermo Fisher Scientific,Inc.) and secondary antibody were sequentially applied on tissue sections at room temperature. The sections were divided into two groups: low HLF expression group ( $\leq$ $60 \%$ ) and high HLF expression group (>60\%). All section slides were assessed independently and blindly by two pathologists.

\section{Western blot analysis}

30 frozen pancreatic tissue samples ( 15 normal and 15 malignant) were obtained. Protein extraction and western blotting were performed as described previously[10]. In brief, 15-80 $\mu \mathrm{g}$ of protein lysates were resolved by electrophoresis on $10 \%$ sodium dodecyl sulfate-polyacrylamide gels. Protein lysates were resoled on $2 \%$ sodium dodecyl sulfate-agarose gel due to its large size, and transferred onto polyvinylidene difluoride membranes. The membranes were followed with overnight incubation with antiHLF antibody (1:2000; Thermo Fisher Scientific,USA) in $4^{\circ} \mathrm{C}$, and incubation with secondary antibody(Thermo Fisher Scientific,USA) for $1 \mathrm{~h}$ in room temperature. Western blots were developed following manufacturer's instructions. The band intensity was determined by relative densitometry using ImageJ (National Institutes of Health) and normalized against the bands obtained for Actin.

\section{Cell culture and Real-Time PCR}

Oral cancer cell line Tca8113 was purchased from ATCC cell bank in the United States and human keratinocytes isolated from normal oral mucosa (HOMK100) was purchased from Cell Research 
Corporation (Singapore). The oral cancer cell lines were were cultured in DMEM/F12 medium (Corning, USA) containing $10 \%$ fetal bovine serum (Gibco, USA) at $37^{\circ} \mathrm{C}$ with $5 \% \mathrm{CO}_{2}$. $\mathrm{HOMK}$ cells were grown in medium supplemented with EpiLife defined growth supplement (Gibco, USA).

Total RNA was extracted from cells by use of the RNeasy Mini Kit (Qiagen), and treated with DNaselRNase free (Invitrogen) and used for cDNA synthesis with oligo(dT)15 primers and SuperScript Reverse Transcriptase (Invitrogen). Expression levels of HLF mRNA was quantified using Taqman probes (Applied Biosystems) with the Taqman gene expression master mix (Applied Biosystems). The gene name and its corresponding Taqman probe used in the Taqman gene expression assays is HLF, Hs00171406_m1. The SDS v2.2 software was used (Applied Biosystems) to analyse the data obtained from the TaqMan Gene Expression Assays.

\section{Result}

Five data series (GSE23558, GSE13601, GSE30784, GSE9844, GSE78060), which were associated with HNSCC, were obtained in NCBI-GEO datasets and analyzed with GEO2R. Based on the threshold of $|\log F C|>2$ and adj.P.value $\leq 0.05$, a total of 17 common HNSCC DEGs were screened, including 11 upregulated DEGs (including RSAD2, MMP1, MMP3, MMP10, IFI6, ISG15, PTHLH, PLAU, MYO1B, CDH3, LAMC2) and 6 downregulated DEGs (including HLF, DPT, ABCA8, CILP, CHRDL1, CFD) (Fig. 1). Differential gene expression was represented as a volcano plot with the distribution of DEGs and as a heat map with hierarchical clustering (Fig. 2).

To test the sensitivity and specificity of HLF as as a prognostic biomarker for HNSCC, an ROC curve analysis was performed, and the AUC was calculated. Area under the curves (AUC) were calculated as follow: 0.9121(GSE9844), 0.9915(GSE13601), 1.0(GSE23558), 0.9196(GSE30784), 0.9196(GSE30784), 0.9519(GSE78060) (Fig. 3).

Although the screening of common DEGs performed in the current study was vigorous and highly reliable, the relative expression levels of HLF in HNSCC were further validated through the online TCGA-based tool GEPIA. The expression levels of HLF in GEPIA demonstrated a consistent trend as the expression profiles, and this result supported the reliability of the data analysis (Fig. 4A). The result of Kaplan-Meier survival analysis suggested that the lower expression level of HLF was negatively correlated with overall survival (Fig. 4B). The datas presented above provided evidence that HLF downregulation might be associated with the initiation or development of pituitary tumours.

Immunohistochemistry staining results confirmed that HLF proteinexpression level is lower than that of matched normal tissue (Fig. 5). HLF protein levels in tumor tissues were significantly lower than that in noraml tissues based on western blot analyses. Western blot results demonstrated that HLF protein levels in tumor tissues were significantly lower than that in noraml tissues $(P<0.05)$, in keeping with the results of the immunohistochemical staining. RT-PCR result validated that HLF mRNA level of Tca8113 cells was significantly lower than that of HOMK cells $(P<0.05)$. 


\section{Discussion}

Hepatic leukaemia factor (HLF) belongs to the the proline and acidic amino acid-rich family and is a transcription factor, which function as circadian modulators [11]. In some cases, this occurs through a direct in-frame joining of E2A exon 12 with HLF exon 4 (type II fusions) [12]. E2A-HLF contributes to leukemogenesis through its potential to inhibit apoptosis [13-14]. Moreover, HLF directly bound to the BS1 site of miR-132 promoter to enhance the expression of miR-132. HLF-mediated miR-132 was able to directly target and inhibit a downstream factor TTK, thus exerting inhibitory effects on glioma cell proliferation, metastasis and radioresistance [15]. Previous studies were showed that Jun is an important oncogene and the expression of the pluripotency factors OCT4 and SOX2 regulate NANOG expression through binding to its promoter [16-19]. Xiang etc [20] demonstrates that HLF transactivates c-Jun to promote tumour initiating cell (TIC) generation and enhances TIC-like properties, thus driving tumour initiation and progression. As well, a positive feedback loop comprising HLF and the pluripotency factors SOX2 and OCT4 may interplays with NANOG to regulate stemness. Recent studies have shown that the pluripotency factors SOX2 and OCT4 and NANOG are involved in its regulation during OSCC development at least to certain degree [21-23]. Therefore, it may be hypothesised that HLF plays a major role in the development of HNSCC. However, Little is known about the relationship between HLF and HNCSS, and this conclusion needs more experimental data to further confirm.

\section{Conclusion}

Through this study, we screened a HNSCC prognostic biomarker HLF by bioinformatics and validated by experiments and clinical data. HLF expression was low in HNSCC tumor cells compared to normal tissues, and low expression of HLF had also been linked to shortened overall survival in patients with HNSCC. However, there are some limitations in this study due to the preset research objectives and limited time. The molecular mechanism of HLF in HNSCC is still not clear, and more research is needed.

\section{Declarations}

\section{Authors' contributions}

WF and XQY initiated and designed the work and prepared the manuscript. DW performed the experiments. JC, WQM, and ZL contributed to the acquisition of patients and tissues specimens and to the analysis and interpretation of data. All authors read and approved the final manuscript.

\section{Funding}

This work was supported by the grant of the Stomatological Hospital of Southern Medical University Projects (NO.PY2020021).

\section{Availability of data and materials}


The data sets during and/or analyzed during the current study are available from the corresponding author on reasonable request.

\section{Ethics approval and consent to participate}

The study was approved by the Stomatological Hospital, Southern Medical University, Guangzhou, China, and all participants were asked to provide signed informed consent to participate in the trial.

\section{Consent for publication}

Not applicable.

\section{Competing interests}

The authors declare that they have no competing interests.

\section{Footnotes}

Publisher's Note

Springer Nature remains neutral with regard to jurisdictional claims in published maps and institutional affiliations.

\section{References}

1. Jemal A, Bray F, Center MM, et al. Global cancer statistics.[J]. CA Cancer J Clin. 2011;61(2):69-90.

2. Surov A, Meyer HJ, Wienke A. Can Imaging Parameters Provide Information Regarding Histopathology in Head and Neck Squamous Cell Carcinoma? A Meta-Analysis.[J].Transl Oncol.2018,11(2):498-503.

3. Posner MR, Hershock DM, Blajman CR, et al. Cisplatin and fluorouracil alone or with docetaxel in head and neck cancer.[J]. N Engl J Med. 2007;357(17):1705-15.

4. Polanska $\mathrm{H}$, Raudenska $\mathrm{M}, \mathrm{Gumulec} J$, et al. Clinical significance of head and neck squamous cell cancer biomarkers.[. J]Oral Oncol. 2014;50(3):168-77.

5. lyengar NM, Ghossein RA, Morris LG, et al. White adipose tissue inflammation and cancer-specific survival in patients with squamous cell carcinoma of. the oral tongue[J]Cancer. 2016;122(24):3794802.

6. Bommi PV, Chand V, Mukhopadhyay NK, et al.NER-factor DDB2 regulates HIF1a and hypoxiaresponse genes in HNSCC.[J].Oncogene.2020,39(8):1784-1796.

7. Zhao L, Chi W, Cao H, et al. Screening and clinical significance of tumor markers in head and neck squamous cell carcinoma through bioinformatics analysis.[. J]Mol Med Rep. 2019;19(1):143-54.

8. Lim AM, Rischin D, Fisher R, et al. Prognostic significance of plasma osteopontin in patients with locoregionally advanced head and neck squamous cell carcinoma treated on TROG 02.02 phase III 
trial.[J]. Clin Cancer Res. 2012;18(1):301-7.

9. Tang Z, Li C, Kang B, et al.GEPIA: a web server for cancer and normal gene expression profiling and interactive analyses.[J]Nucleic Acids Res.2017,45(W1):W98-102.

10. Singh AP, et al. Inhibition of MUC4 expression suppresses pancreatic tumor cell growth and metastasis. Cancer Res. 2004;64:622-30.

11. Inaba T, Shapiro LH, Funabiki T, et al. DNA-binding specificity and trans-activating potential of the leukemia-associated E2A-hepatic leukemia factor fusion protein.[J]. Mol Cell Biol. 1994;14(5):340313.

12. Hunger SP, Devaraj PE, Foroni $L$, et al.Two types of genomic rearrangements create alternative E2AHLF fusion proteins in t(17;19)-ALL.[J].Blood.1994,83(10):2970-7.

13. Inukai T, Inaba T, Dang J, et al. TEF, an antiapoptotic bZIP transcription factor related to the oncogenic E2A-HLF chimera, inhibits cell growth by down-regulating expression of the common beta chain of cytokine receptors. [J]Blood. 2005;105(11):4437-44.

14. Hunger SP, Li S, Fall MZ, et al.The proto-oncogene HLF and the related basic leucine zipper protein TEF display highly similar DNA-binding and transcriptional regulatory properties. [J].Blood.1996,87(11):4607-17.

15. Chen $\mathrm{S}$, Wang $\mathrm{Y}, \mathrm{Ni}$ C, et al. HLF/miR-132/TTK axis regulates cell proliferation, metastasis and radiosensitivity of glioma cells.[. J]Biomed Pharmacother. 2016;83:898-904.

16. Maeda S, Karin M. Oncogene at last-c-Jun promotes liver cancer in mice.[. J]Cancer Cell. 2003;3(2):102-4.

17. Takeda D, Hasegawa T, Ueha T, et al. Induced Pluripotent-stem-cell Related Genes Contribute to Dedifferentiation in. Oral Squamous Cell Carcinoma[J]Anticancer Res. 2017;37(3):1075-82.

18. Yu HH, Featherston T, Tan ST, et al.Characterization of Cancer Stem Cells in Moderately Differentiated Buccal Mucosal Squamous Cell Carcinoma.[J].Front Surg.2016,3:46.

19. Ram R, Brasch HD, Dunne JC, et al.The Identification of Three Cancer Stem Cell Subpopulations within Moderately Differentiated Lip Squamous Cell Carcinoma.[J].Front Surg.2017,4:12.

20. Xiang DM, Sun W, Zhou T, et al.Oncofetal HLF transactivates c-Jun to promote hepatocellular carcinoma development and sorafenib resistance.[J].Gut.2019,68(10):1858-1871.

21. Grubelnik G, Boštjančič E, Grošelj A, et al.Expression of NANOG and Its Regulation in Oral Squamous Cell Carcinoma.[J].Biomed Res Int.2020,2020:8573793.

22. Chien CS, Wang ML, Chu PY, et al.Lin28B/Let-7 Regulates Expression of Oct4 and Sox2 and Reprograms Oral Squamous Cell Carcinoma Cells to a Stem-like State.[J].Cancer Res.2015,75(12):2553-65.

23. Vijayakumar G, Narwal A, Kamboj M, et al.Association of SOX2, OCT4 and WNT5A Expression in Oral Epithelial Dysplasia and Oral Squamous Cell Carcinoma: An Immunohistochemical Study.[J].Head Neck Pathol.2020,14(3):749-757. 
Figures

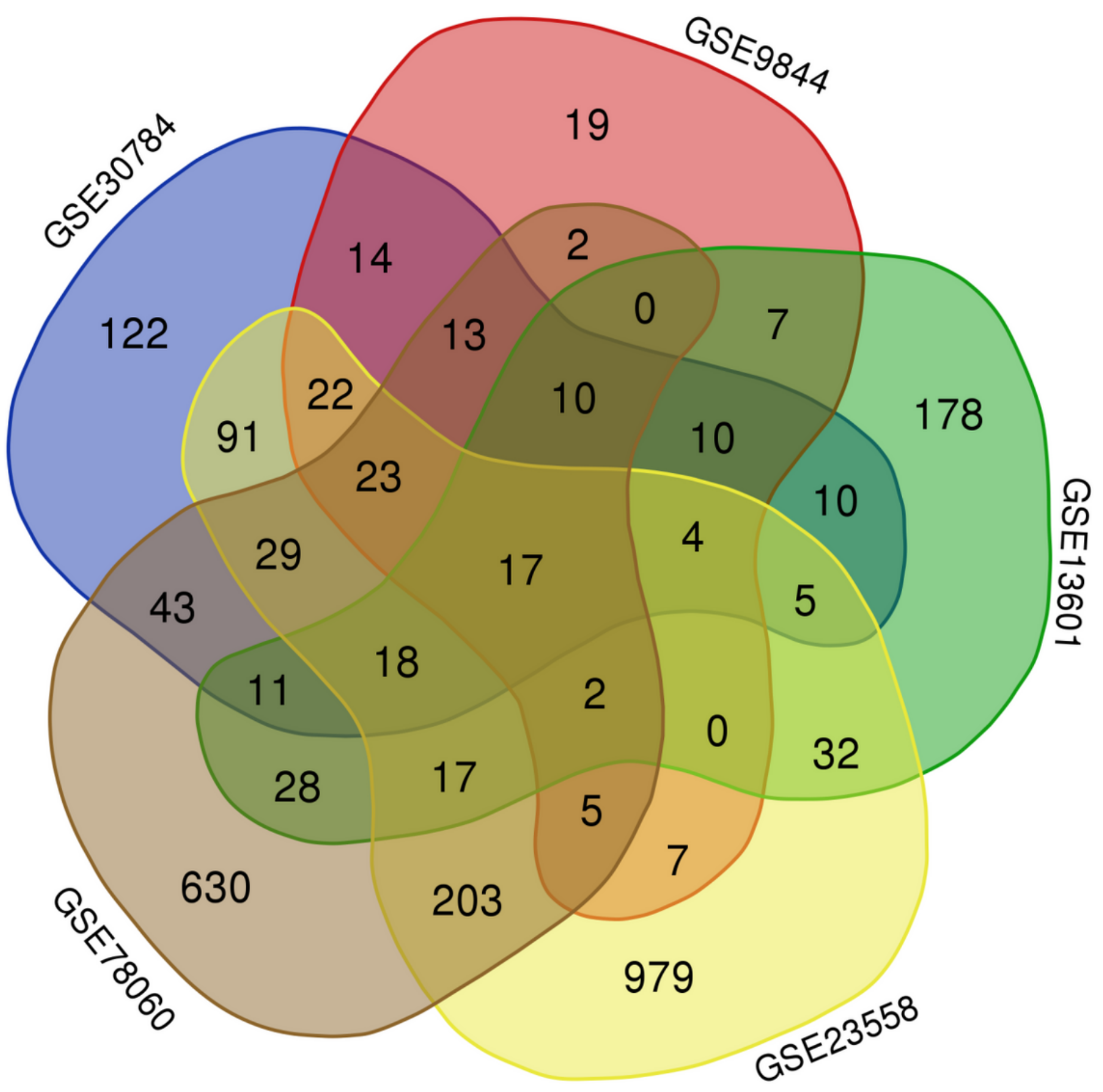

Figure 1

A total of 17 common HNSCC DEGs were screened based on the data of the five HNSCC gene expression series from GEO datasets. 

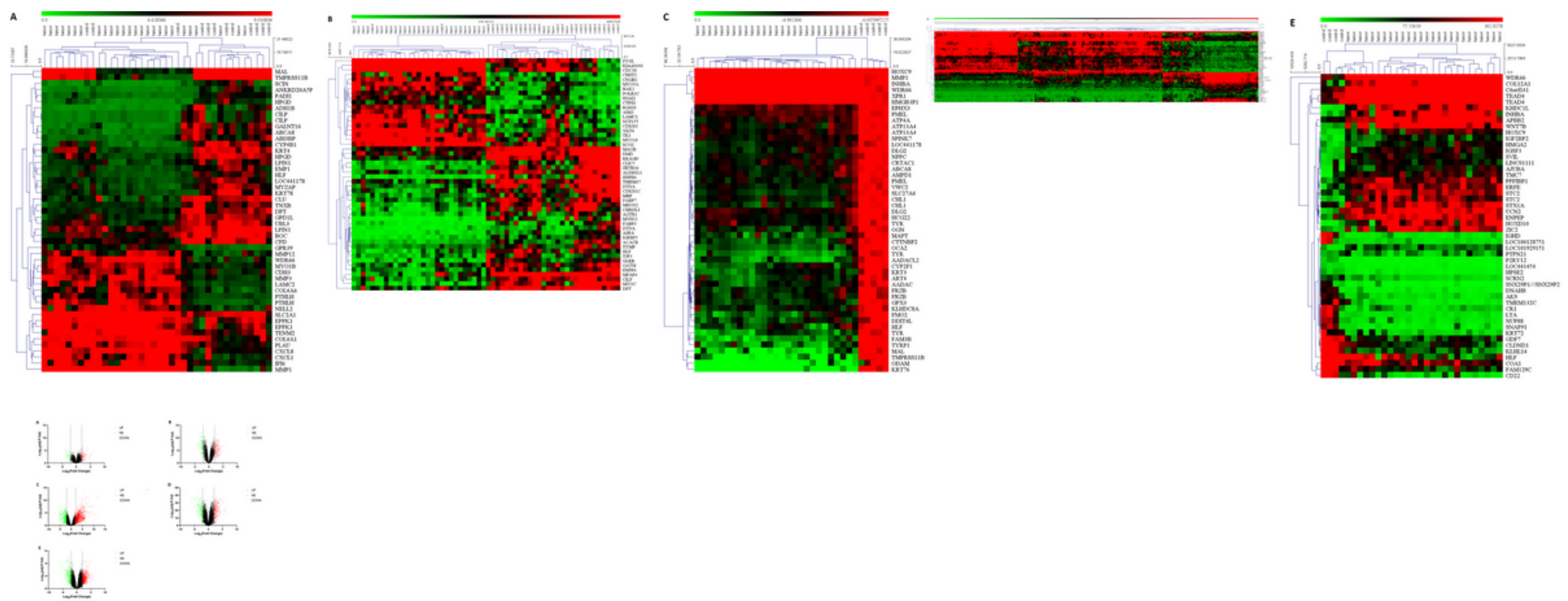

Figure 2

Visual presentation of the differentially expressed genes ( $|\log \mathrm{FC}| \geq 2 ; \mathrm{P} \leq 0.05)$ included in the three data series examined in the present study. Hierarchical clustering graphs of genes in the (A)GSE9844区(B) GSE13601』(C) GSE23558®(D)GSE30784『(E) GSE78060 data series are shown. Red and green represent upregulated and downregulated genes in cancerous tissues compared with normal samples, respectively.

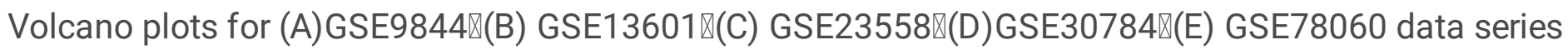
are displayed. The $x$-axis represents the log2 of Fold Change and the $y$-axis represents the negative log 10 of the P-value. Red and grey foci on the graphs represent differently and non-differentially expressed mRNAs, respectively, in head and neck squamous cell carcinoma. 

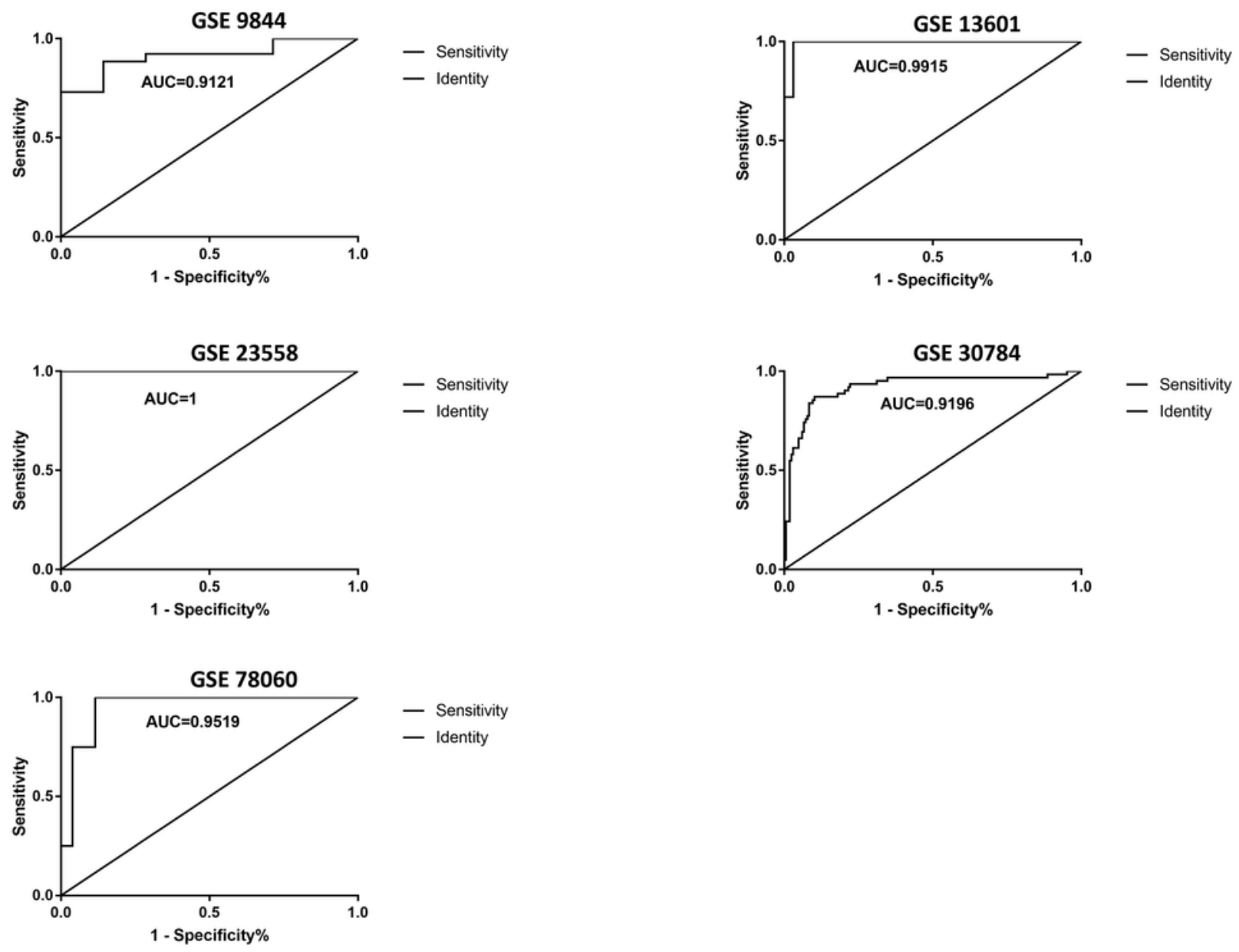

Figure 3

ROC curve validated the sensitively, specificity of HLF as a predictive biomarker for HNSCC prognosis. 

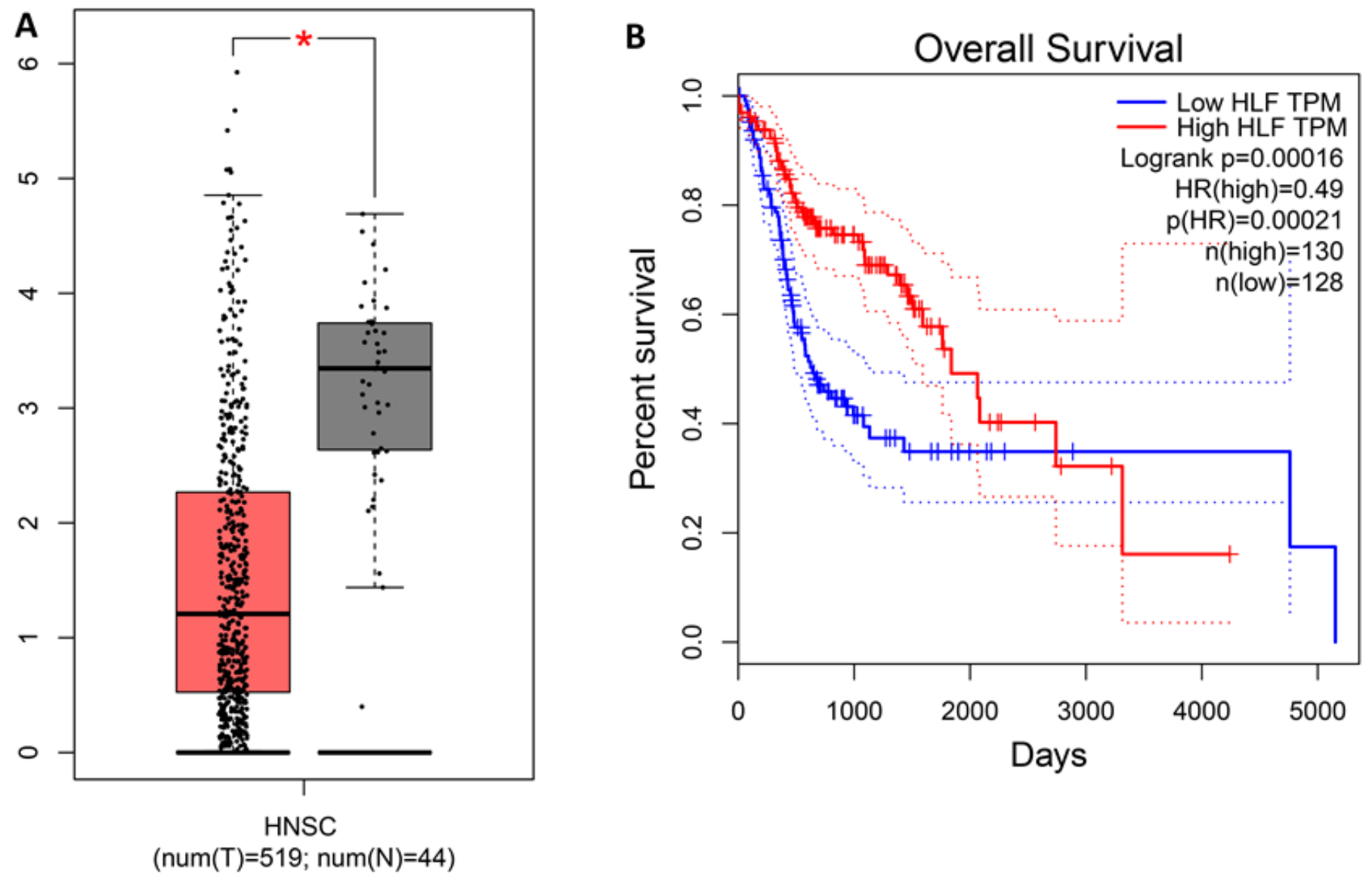

\section{Figure 4}

The relative expression levels of HLF in HNSCC were further validated through the online TCGA-based tool GEPIA. (A) Relative expression levels of HLF in HNSCC; (B) K-M curves showed that lower expression level of SFRP1 was signifificantly related to poor prognosis of HNSCC patients.
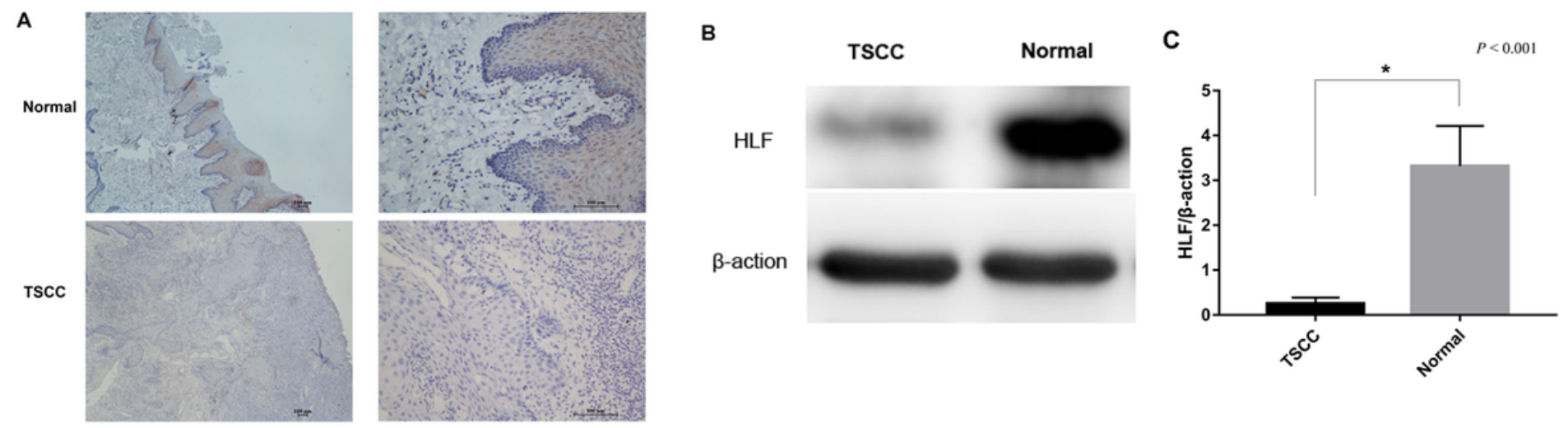

Figure 5

The protein expression of HLF in TSCC and matched normal tissues. (A) Immunohistochemical analysis revealed lower expression levels of HLF in TSCC compared to normal controls. (B) (C) Western blot 
analysis demonstrated reduced protein levels of HLF in TSCC tissues, compared with matched normal tissues.

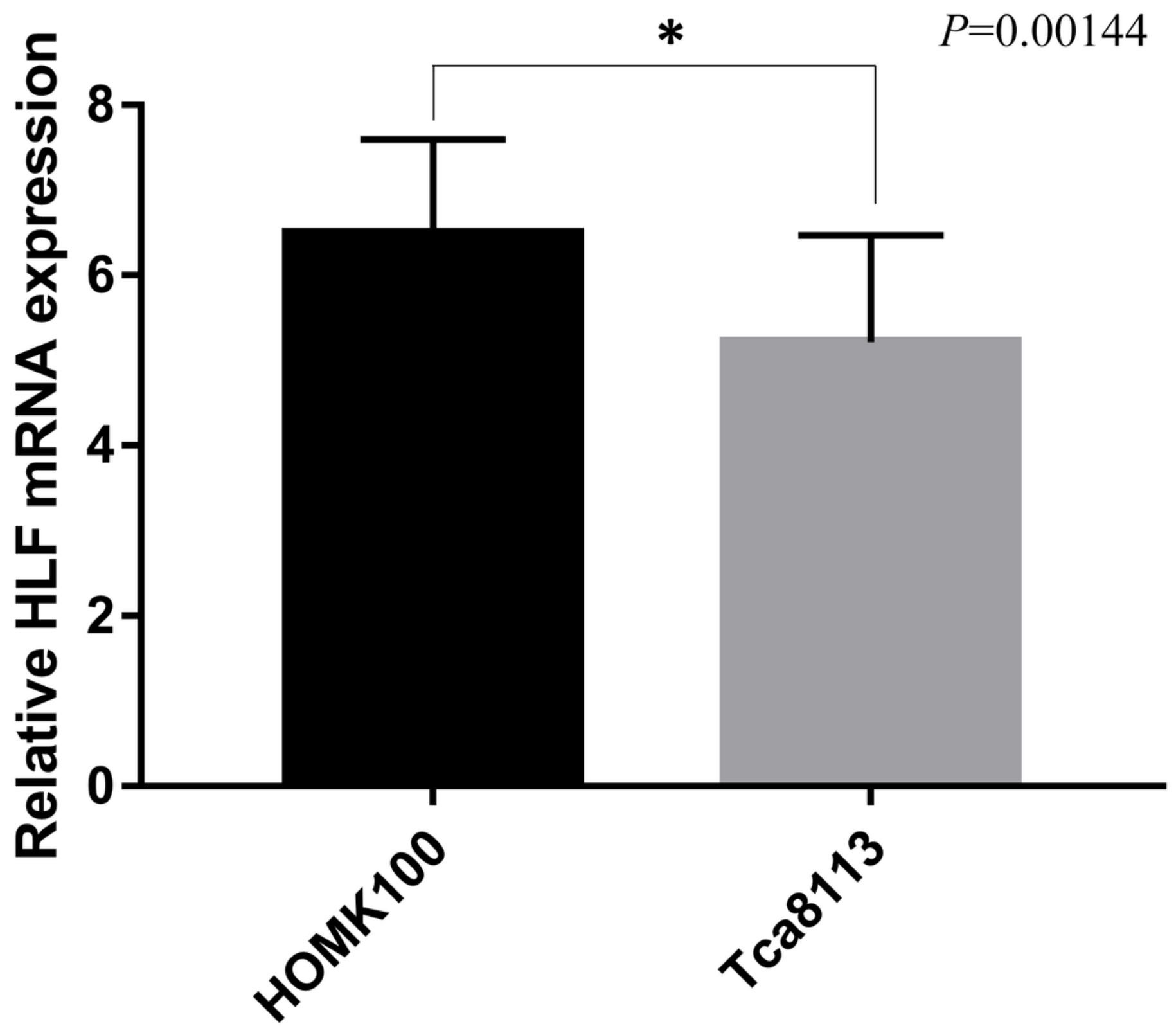

Figure 6

RT-PCR result showed that The expression levels of HLF mRNA in Tca8113 cells was lower than that in HOMK cells. 